Original Research Paper

\title{
The Regularity of the Solutions to the Cauchy Problem for the Quasilinear Second-Order Parabolic Partial Differential Equations
}

\author{
Mykola Yaremenko \\ Partial Differential Equation, KPI, Kyiv, Ukraine
}

Article history

Received: 01-04-2020

Revised: 07-05-2020

Accepted: 29-05-2020

Email: math.kiev@gmail.com

\begin{abstract}
This article is dedicated to expanding our comprehension of the regularity of the solutions to the Cauchy problem for the quasilinear second-order parabolic partial differential equations under fair general conditions on the nonlinear perturbations. In this paper have been obtained that the sequence of the weak solutions $u^{z} \in V_{1,0}{ }^{2}, z=1,2, \ldots$. to the Cauchy problems for the Equations (15) under the initial conditions $u^{z}$ $(0, x)=\varphi_{0}{ }^{z}$ converges to the weak solution to the Cauchy problem for the Equation (1) under the initial condition $u(0, x)=u_{0}$ in $V_{1,0}{ }^{2}$.
\end{abstract}

Keywords: Quasi-Linear Partial Differential Equations, Nonlinear Partial Differential Equations, Parabolic, Nonlinear Operator, Weak Solution, A Priori Estimations

\section{Introduction}

Let us consider the quasilinear second-order parabolic partial differential equations:

$$
\begin{aligned}
& \frac{\partial}{\partial t} u+\lambda u-\sum_{i, j=\mathbf{1}, ., l} \frac{\partial}{\partial x_{i}}\left(a_{i j}(t, x, u) \frac{\partial}{\partial x_{j}} u\right), \\
& +b(t, x, u, \nabla u)=f(t, x),
\end{aligned}
$$

under the initiation conditions:

$$
u(\mathbf{0}, x)=u_{0}(x)
$$

where the $u(t, x)$ is the unknown function, $\lambda>0$ is a real number and $f(t, x)=f$ is a given function. The term $b(t, x$, $u, \nabla u)$ is a measurable function of four arguments.

The matrix $a_{i j}(t, x, u)$ is a measurable elliptical matrix $l$ $\times l$ size such that there is a number $v: \quad 0<v<\infty$ and:

$$
v \sum_{i=1}^{l} \xi_{i}^{2} \leq \sum_{i j=1, \ldots, l} a_{i j}(t, x, u) \xi_{i} \xi_{j} \quad \forall \xi \in R^{l}
$$

for almost every $t \in[\mathbf{0}, T]$ and $x \in R^{l}$. Or we will consider a more restrictive condition:

$$
v \sum_{i=1}^{l} \xi_{i}^{2} \leq \sum_{i j=1, \ldots, l} a_{i j}(t, x, u) \xi_{i} \xi_{j} \leq \mu \sum_{i=1}^{l} \xi_{i}^{2} \forall \xi \in R^{l}
$$

\section{Definition}

A real-valued function $u(t, x)$ is called a weak solution to the parabolical partial differential Equation (1) if the integral identity:

$$
\begin{aligned}
& \left.\langle u(\tau), v(\tau)\rangle\right|_{0} ^{t}+ \\
& +\int_{0}^{t}\left(-\left\langle u(\tau), \partial_{t} v(\tau)\right\rangle+\lambda\langle u(\tau), v(\tau)\rangle\right) d \tau+ \\
& +\int_{0}^{t}\left\langle\sum_{i, j=1, \ldots, l} a_{i j} \frac{\partial}{\partial x_{j}} u, \frac{\partial}{\partial x_{i}} v\right\rangle d \tau+ \\
& +\int_{0}^{t}\langle b, v\rangle d \tau=\int_{0}^{t}\langle f, v\rangle d \tau
\end{aligned}
$$

holds for almost every $t \in[\mathbf{0}, T], x \in R^{l}$ and for all $v \in W_{\mathbf{1 , 0}}^{q}$.

The main object of this paper is the regularity properties of the solutions to the quasilinear parabolical partial differential Equation (1) under the conditions that its coefficients belong to the certain functional classes and functional spaces.

The conditions of linear growth:

1. $b(t, x, y, z)$ is a measurable function of its arguments and $b \in L_{l o c}^{1}\left(R^{l}\right)$

2. Function $b(t, x, y, z) t \in[\mathbf{0}, T]$ satisfies inequality:

$$
|b(t, x, u, \nabla u)| \leq \mu_{1}(x)|\nabla u|+\mu_{2}(x)|u|+\mu_{3}(x)
$$


for almost everywhere and almost every $t \in[\mathbf{0}, T]$, where the functions $\mu_{1}^{2} \in P K_{\beta}(A), \mu_{2} \in P K_{\beta}(A)$ and $\mu_{3} \in L^{p}\left(R^{l}\right)$.

3. The increase of function $b(t, x, y, z)$ satisfies the inequality:

$$
\begin{aligned}
& |b(t, x, u, \nabla u)-b(t, x, v, \nabla v)| \leq \\
& \leq \mu_{4}(x)|\nabla(u-v)|+\mu_{5}(x)|u-v|
\end{aligned}
$$

almost everywhere and almost every $t \in\left[\begin{array}{ll}\mathbf{0}, T \\ \text {, }\end{array}\right.$ where the functions $\mu_{4}^{2} \in P K_{\beta}(A), \mu_{5} \in P K_{\beta}(A)$.

Here we introduce the class of form-bounded functions $P K_{\beta}$ according to formula-definition:

$$
P K_{\beta}(A)=\left\{\begin{array}{l}
g \in L_{l o c}^{1}\left(R^{l}, d^{l} x\right):\left|\left\langle g|h|^{2}\right\rangle\right| \leq \\
\leq \beta\left\langle A^{\frac{1}{2}} h, A^{\frac{1}{2}} h\right\rangle+c(\beta)\|h\|_{2}^{2}
\end{array}\right\},
$$

where a $h \in D\left(A^{\frac{1}{2}}\right)$ and $\beta>0$ is a form-boundary and $c(\beta)$ $\in R^{1}$

The general information on the partial differential equations and the existence of their solutions can be found in the extensive literature on the conditions on their coefficients under which there are the solutions of these equations in a specific functional space (Adams and Hedberg, 1996; Gilbarg and Trudinger, 1983; Ladyzenskaja et al., 1968; Nirenberg, 1994; Veron, 1996; Yaremenko, 2017a; 2017b). O. Ladyzhenskaya, N. Uraltseva, O.A. Solonnikov developed the Ennio de Giorgi's method (DeGiorgi, 1968) for establishing a priory estimation of the solution of such equations. 1960 J. Moser enhance the maximum principle and created a new method of studying the regularity of the solutions of elliptic differential equations and Harnack's inequality under the assumption that the coefficients are bounded measurable and satisfy a uniform ellipticity condition, these results were summarized in the work of Ladyzenskaja et al. (1968).

A Lebesgue space $L^{p}\left(R^{l}, d^{l} x\right)$ for $1<p<\infty$ can be defined as a set of all real-valued measurable functions defined almost everywhere such that the Lebesgue integral of its absolute value raised to the $p$-th power is a finite number with its natural norm:

$$
\begin{aligned}
& \|u\|_{L^{p}}=\left(\int\left|u\left(x_{1}, \ldots, x_{n}\right)\right|^{p} d^{l} x\right)^{\frac{1}{p}} \\
& =\left(\int_{R^{l}}|u(x)|^{p} d^{l} x\right)^{\frac{1}{p}}=\left\langle|u|^{p}\right\rangle^{\frac{1}{p}}
\end{aligned}
$$

The dual or adjoint space of $L^{p}\left(R^{l}, d^{l} x\right)$ for $1<p<\infty$ has a natural isomorphism with $L^{q}\left(R^{l}, d^{l} x\right)$, where $\frac{1}{p}+\frac{1}{q}=1$ or $q=\frac{p}{p-1}$.

We will use the inequality:

$$
\langle f, g\rangle \leq\|f\|_{p}\|g\|_{q} \leq \frac{\varepsilon^{p}}{p}\|f\|_{p}^{p}+\frac{\mathbf{1}}{\varepsilon^{q} q}\|g\|_{q}^{q},
$$

where $f \in L^{p}\left(R^{l}\right), g \in L^{q}\left(R^{l}\right), \varepsilon>\mathbf{0}$ and its consequence:

$$
\begin{aligned}
& \left\langle f, f|f|^{p-2}\right\rangle=\|f\|_{L^{p}\left(R^{l}\right)}\left\|f|f|^{p-2}\right\|_{L^{q}\left(R^{l}\right)} \\
& =\frac{\mathbf{1}}{p}\|f\|_{L^{p}\left(R^{l}\right)}^{p}+\frac{\mathbf{1}}{q}\left\|f|f|^{p-2}\right\|_{L^{q}\left(R^{l}\right)}^{p-\mathbf{1}}=\|f\|_{L^{p}\left(R^{l}\right)}^{p},
\end{aligned}
$$

The $f \in L^{p}$ yields $f|f|^{p-2} \in L^{q}$ that justify the last equation (Gilbarg and Trudinger, 1983; Ladyzenskaja et al., 1968).

Let us denote $W_{k}^{p}\left(R^{l}, d^{l} x\right)$ given Sobolev space for $1<p<\infty$ with a natural norm:

$$
\begin{aligned}
& \|u\|_{W_{k}^{p}}=\left(\sum_{i=\mathbf{0}}^{k} \int\left|u^{(i)}\left(x_{1}, \ldots, x_{n}\right)\right|^{p} d^{l} x\right)^{\frac{1}{p}} \\
& =\left(\|u\|_{p}^{p}+\sum_{1 \leq s \mid \leq m}\left\|D^{s} u\right\|^{p}\right)^{\frac{1}{p}}=\left(\sum_{i=\mathbf{0}}^{k}\left\|u^{(i)}\right\|_{p}^{p}\right)^{\frac{1}{p}} .
\end{aligned}
$$

The dual space of $W_{k}^{p}\left(R^{l}, d^{l} x\right)$ for $1<p<\infty$ is $W_{-k}^{q}\left(R^{l}, d^{l} x\right)$ and the dual space of $W_{-k}^{p}\left(R^{l}, d^{l} x\right)$ for $1<p$ $<\infty$ and $\frac{\mathbf{1}}{p}+\frac{\mathbf{1}}{q}=\mathbf{1}$ is $W_{k}^{q}\left(R^{l}, d^{l} x\right)$, Sobolev spaces are reflexive (Fijavz et al., 2007).

Let us consider a linear parabolic equation as an exemplar:

$$
L u=\left[\begin{array}{l}
\frac{\partial}{\partial_{t}}-\sum_{i, k=1, \ldots, l} a_{k j}(t, x) \nabla_{k} \nabla_{j}- \\
-\sum_{k=1, \ldots, l} b_{k}(t, x) \nabla_{k}
\end{array}\right] u(t, x)=\mathbf{0}
$$

under the conditions $\exists v, \mu: \quad \mathbf{0}<v \leq \mu<\infty$ such that:

$$
v \sum_{i=1}^{l} \xi_{i}^{2} \leq \sum_{i j=\mathbf{1}, \ldots, l} a_{i j}(t, x) \xi_{i} \xi_{j} \leq \mu \sum_{i=1}^{l} \xi_{i}^{2}
$$

and linear perturbation-potential $b_{k}(t, x): R^{l} \mapsto R^{l}$.

In traducing the notations: 


$$
\begin{gathered}
\nabla \circ a \circ \nabla u=\sum_{i, j=1, \ldots, l} \frac{\partial}{\partial x_{i}} a_{i j} \frac{\partial}{\partial x_{j}} u, \\
b \nabla u=b \circ \nabla u=\sum_{i=1, \ldots, l} b_{i} \frac{\partial}{\partial x_{i}} u
\end{gathered}
$$

and assuming $b \circ a^{-1} \circ b \in P K_{\beta}(A)$ for some $\beta<1$ we obtain:

$$
|\langle\nabla h \circ b h\rangle| \leq \sqrt{\beta}\langle A h, h\rangle+c(\beta) \frac{\mathbf{1}}{\mathbf{2} \sqrt{\beta}}\|h\|_{2}^{2},
$$

according to the KLMN-theorem, there is a preserving $C_{0^{-}}$semigroups of $L^{\infty}$ - contraction $e^{-t \Lambda_{n}}, \frac{\mathbf{2}}{\mathbf{2}-\sqrt{\beta}} \leq n \leq \infty$ such that $\Lambda_{2}=A+b \circ \nabla$. Assuming $A$ is Laplace operator $A=\Delta$ we are obtaining an estimation:

$$
|\langle\nabla h \cdot b h\rangle| \leq \sqrt{\beta}\|\nabla h\|^{2}+\frac{c(\beta)}{2 \beta}\|h\|^{2} \quad \forall h \in D(\Delta) .
$$

The operator $B_{1}=\nabla \circ b$ of the domain $D\left(B_{1}\right)=\left\{u \in L^{1} ;|\nabla u| \in L_{l o c}^{1} ; b \circ \nabla u \in L^{1}\right\} \quad$ is $A_{1}$-bunded with relative bound zero namely $D\left(B_{1}\right) \supset D\left(A_{1}\right)$ and:

$$
\left\|B_{1} h\right\|_{1} \leq \alpha\left\|A_{1} h\right\|_{1}+k(\alpha)\|h\|_{1}, \quad h \in D\left(A_{1}\right)
$$

holds for all $\alpha>0$ and $k(\alpha)<\infty$. There are $s>0$ and $\beta(s)$ $<1$ such that $\int_{0}^{s}\left\|B_{1} e^{-t A_{1}} h\right\|_{1} d t \leq \beta(\mathrm{s})\|h\|_{1}, \quad h \in D\left(A_{1}\right)$. The operator $A_{1}+B_{1}$ of the domain $D\left(A_{1}\right)$ generates $C_{0^{-}}$ semigroup $T_{1}^{t}$ consistent with $T^{t}=\exp (-t(A+b \circ \nabla))$ such that $\left\|T_{1}^{t}\right\|_{1 \rightarrow 1} \leq \frac{1}{1-\beta(\mathrm{s})} \exp \left(-t \frac{\log (\mathbf{1}-\beta(\mathrm{s}))}{s}\right), t>\mathbf{0}$.

\section{The Estimation of the Solutions to the Equation (1)}

For almost every $t \in[0, T]$, let us consider the integral identity:

$$
\begin{aligned}
& \left.\langle u(\tau), v(\tau)\rangle\right|_{0} ^{t}+\int_{0}^{t}\left(-\left\langle u(\tau), \partial_{t} v(\tau)\right\rangle+\lambda\langle u(\tau), v(\tau)\rangle\right) d \tau \\
& +\int_{0}^{t}\left\langle\sum_{i, j=1, \ldots, l} a_{i j} \frac{\partial}{\partial x_{j}} u, \frac{\partial}{\partial x_{i}} v\right\rangle d \tau+\int_{0}^{t}\langle b, v\rangle d \tau=\int_{0}^{t}\langle f, v\rangle d \tau,
\end{aligned}
$$

where functions $u(t, x) \in W_{\mathbf{1 , 0}}^{p}$ and $v \in W_{\mathbf{1}, \mathbf{0}}^{q}$.

For $t \in[0, T]$ identity (6) can be rewritten as:

$$
\begin{aligned}
& \left.\langle u(\tau), v(\tau)\rangle\right|_{0} ^{t} \\
& +\int_{0}^{t}\left(-\left\langle u(\tau), \partial_{\tau} v(\tau)\right\rangle+\left\langle\sum_{i, j=1, \ldots, l} a_{i j} \frac{\partial}{\partial x_{j}} u, \frac{\partial}{\partial x_{i}} v\right\rangle d \tau\right. \\
& =\int_{0}^{t}\langle f, v\rangle d \tau-\int_{0}^{t}(\lambda\langle u(\tau), v(\tau)\rangle) d \tau-\int_{0}^{t}\langle b, v\rangle d \tau
\end{aligned}
$$

Let us put $v(\tau)=u|u|^{p-2}(\tau)$ and estimate:

$$
\begin{aligned}
& \left.\left\langle u(\tau), u|u|^{p-2}(\tau)\right\rangle\right|_{0} ^{t}+\lambda \int_{0}^{t}\left|\left\langle u(\tau), u|u|^{p-2}(\tau)\right\rangle\right| d \tau
\end{aligned}
$$

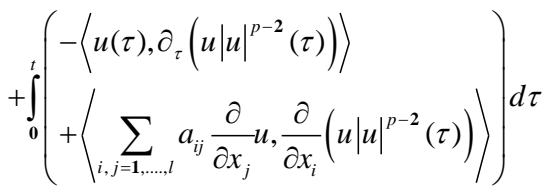

$$
\begin{aligned}
& \leq \int_{0}^{t}\left|\left\langle f,\left(u|u|^{p-2}(\tau)\right)\right\rangle\right| d \tau \\
& +\int_{0}^{t}\left\{\begin{array}{l}
\mu_{1}(t, x)|\nabla u|+\mu_{2}(t, x)|u|+ \\
+\mu_{3}(t, x), u|u|^{p-2}(\tau)
\end{array}\right\rangle d \tau .
\end{aligned}
$$

From (1) under the conditions (4) we obtain (6). Next, we estimate every term separately:

$$
\begin{aligned}
& \left|\left\langle f, u|u|^{p-2}(\tau)\right\rangle\right| \leq\|f\|\left|\left\|u|u|^{p-2}(\tau)\right\|\right. \\
& \leq \frac{\sigma^{p}}{p}\|f\|^{p}+\frac{\mathbf{1}}{q \sigma^{q}}\left\|u|u|^{p-2}(\tau)\right\|^{q}, \\
& \left\langle\sum_{i, j=\mathbf{1}, \ldots, l} a_{i j} \frac{\partial}{\partial x_{j}} u, \frac{\partial}{\partial x_{i}}\left(u|u|^{p-2}(\tau)\right)\right\rangle= \\
& =\frac{\mathbf{4}(p-\mathbf{1})}{p^{\mathbf{2}}}\left\langle\nabla\left(u|u|^{\frac{p-2}{2}}(\tau)\right) \circ a \circ \nabla\left(u|u|^{\frac{p-2}{2}}(\tau)\right)\right\rangle,
\end{aligned}
$$

denoting $w=u|u|^{\frac{p-2}{2}}(\tau)$ and $\nabla w=\frac{p}{2}|u|^{\frac{p-2}{2}} \nabla u$ :

$$
\begin{aligned}
& \left\langle\mu_{1}|\nabla u|,|u|^{p-1}\right\rangle=\left\langle\mu_{1}|u|^{\frac{p-2}{2}}|\nabla u|,|u|^{\frac{p}{2}}\right\rangle \leq \frac{2}{p}\left\langle\mu_{1}|\nabla w|,|w|\right\rangle, \\
& \left\langle\mu_{2}(x), w^{2}\right\rangle \leq \beta\langle\nabla w \circ a \circ \nabla w\rangle+c(\beta)\|w\|^{2}, \\
& \left\langle\mu_{3}(x),|u|^{p-1}\right\rangle \leq\left\|\mu_{3}\right\|\left\||u|^{p-1}\right\|=\left\|\mu_{3}\right\|\|u\|^{p-1}
\end{aligned}
$$

Applying a form-boundary condition to $\frac{\mathbf{2}}{p}\left\langle\mu_{1}|\nabla w|,|w|\right\rangle$ $\leq \frac{\mathbf{2}}{p}\left\|\mu_{1} w\right\|\|\nabla w\|$, we have:

$$
\left\|\mu_{1} w\right\|=\left\langle\left(\mu_{1} w\right)^{2}\right\rangle^{\frac{1}{2}} \leq\left(\beta\langle\nabla w \circ a \circ \nabla w\rangle+c(\beta)\|w\|^{2}\right)^{\frac{1}{2}},
$$

using Young and Holder inequalities are obtaining: 


$$
\begin{aligned}
& \frac{\mathbf{2}}{p}\left\langle\mu_{1}|\nabla w|,|w|\right\rangle \leq \frac{\mathbf{2}}{p}\left\|\mu_{1} w\right\|\|\nabla w\|=\frac{\mathbf{2}}{p}\|\nabla w\|\left\langle\left(\mu_{1} w\right)^{2}\right\rangle^{\frac{\mathbf{1}}{2}} \\
& \leq \frac{\mathbf{2}}{p}\|\nabla w\|\left(\beta\langle\nabla w \circ a \circ \nabla w\rangle+c(\beta)\|w\|^{2}\right)^{\frac{\mathbf{1}}{2}} \\
& \leq \frac{\mathbf{1}}{p}\left(\frac{\mathbf{1}}{\varepsilon^{2}}\|\nabla w\|^{2}+\varepsilon^{2}\left(\beta\langle\nabla w \circ a \circ \nabla w\rangle+c(\beta)\|w\|^{2}\right)\right) \\
& \leq \frac{\mathbf{1}}{p}\left(\frac{\mathbf{1}}{\varepsilon^{2}}\langle\nabla w \circ a \circ \nabla w\rangle+\varepsilon^{2}\left(\beta\langle\nabla w \circ a \circ \nabla w\rangle+c(\beta)\|w\|^{2}\right)\right) .
\end{aligned}
$$

Thus, we have obtained an estimation:

$\int_{0}^{t}\left(\left\langle\partial_{\tau} u(\tau),\left(u|u|^{p-2}(\tau)\right)\right\rangle+\left\langle\sum_{i, j=1, \ldots, l} a_{i j} \frac{\partial}{\partial x_{j}} u, \frac{\partial}{\partial x_{i}}\left(u|u|^{p-2}(\tau)\right)\right\rangle\right) d \tau$

$+\lambda \int_{0}^{t}\left|\left\langle u(\tau), u|u|^{p-2}(\tau)\right\rangle\right| d \tau+$

$\leq \int_{0}^{t}\left|\frac{\sigma^{p}}{p}\|f\|^{p}+\frac{1}{q \sigma^{q}}\left\|u|u|^{p-2}(\tau)\right\|^{q}\right| d \tau$

$+\int_{0}^{t}\left(\frac{\mathbf{1}}{p}\left(\frac{\mathbf{1}}{\varepsilon^{2}}\|\nabla w\|^{2}+\varepsilon^{2}\left(\beta\langle\nabla w \circ a \circ \nabla w\rangle+c(\beta)\|w\|^{2}\right)\right)(\tau)\right) d \tau$

$+\int_{0}^{t}\left(\beta\langle\nabla w \circ a \circ \nabla w\rangle+c(\beta)\|w\|^{2}+\frac{\mathbf{1}}{\gamma^{q} q}\|w\|^{2}+\frac{\gamma^{p}}{p}\left\|\mu_{3}\right\|^{p}\right) d \tau$.

For almost all $t$ applying $\left.\left\langle u(\tau), u|u|^{p-2}(\tau)\right\rangle\right|_{0} ^{t}$ $=p \int_{0}^{t}\left\langle\partial_{\tau} u(\tau),\left(u|u|^{p-2}(\tau)\right)\right\rangle d \tau$ we have had:

$$
\begin{aligned}
& \left.\frac{\mathbf{1}}{p}\|w\|^{2}\right|_{0} ^{t}+\mathbf{4} \frac{p-\mathbf{1}}{p^{2}} \int_{0}^{t}(\langle\nabla w \circ a \circ \nabla w\rangle) d \tau+\lambda \int_{0}^{t}\|w\|^{2} d \tau \\
& \leq \int_{0}^{t}\left|\frac{\sigma^{p}}{p}\|f\|^{p}+\frac{\mathbf{1}}{q \sigma^{q}}\left\|u|u|^{p-2}(\tau)\right\|^{q}\right| d \tau
\end{aligned}
$$

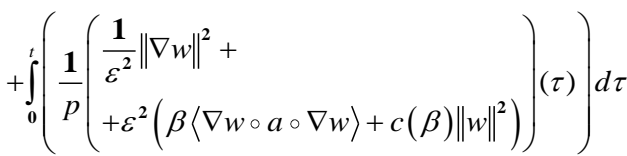

$$
\begin{aligned}
& +\int_{0}^{t}\left(\begin{array}{l}
\beta\langle\nabla w \circ a \circ \nabla w\rangle+c(\beta)\|w\|^{2}+ \\
+\frac{\mathbf{1}}{\gamma^{q} q}\|w\|^{2}+\frac{\gamma^{p}}{p}\left\|\mu_{3}\right\|^{p}
\end{array}\right) d \tau .
\end{aligned}
$$

Since $\left\|u|u|^{p-2}(\tau)\right\|^{q}=\left\langle|u|^{(p-1) q}\right\rangle=\|u\|^{p}=\|w\|^{2}$ we obtain:

$$
\begin{aligned}
& \left.\frac{\mathbf{1}}{p}\|w\|^{2}\right|_{0} ^{t}+\mathbf{4} \frac{p-\mathbf{1}}{p^{2}} \int_{0}^{t}\langle\nabla w \circ a \circ \nabla w\rangle d \tau+\lambda \int_{0}^{t}\|w\|^{2} d \tau \\
& \leq\left(\frac{\mathbf{1}}{q \sigma^{q}}+\frac{c(\beta)}{p} \varepsilon^{2}+c(\beta)+\frac{\mathbf{1}}{q \gamma^{q}}\right) \int_{0}^{t}\|w\|^{2} d \tau \\
& +\left(\frac{\mathbf{1}}{p}\left(\frac{\mathbf{1}}{\varepsilon^{2}}+\beta \varepsilon^{2}\right)+\beta\right) \int_{0}^{t}\langle\nabla w \circ a \circ \nabla w\rangle d \tau \\
& +\int_{0}^{t} \frac{\sigma^{p}}{p}\|f\|^{p} d \tau+\int_{0}^{t} \frac{\gamma^{p}}{p}\left\|\mu_{3}\right\|^{p} d \tau .
\end{aligned}
$$

In case of $p=2$ there is the next estimation:

$$
\begin{aligned}
& \left.\frac{\mathbf{1}}{\mathbf{2}}\|u\|^{2}\right|_{0} ^{t}+\int_{0}^{t}\langle\nabla u \circ a \circ \nabla u\rangle d \tau+\lambda \int_{0}^{t}\|u\|^{2} d \tau \\
& \leq\left(\frac{\mathbf{1}}{\mathbf{2} \sigma^{2}}+\frac{c(\beta)}{\mathbf{2}} \varepsilon^{2}+c(\beta)+\frac{\mathbf{1}}{\mathbf{2} \gamma^{2}}\right)_{0}^{t}\|u\|^{2} d \tau \\
& +\left(\frac{\mathbf{1}}{\mathbf{2}}\left(\frac{\mathbf{1}}{\varepsilon^{2}}+\beta \varepsilon^{2}\right)+\beta\right) \int_{0}^{t}\langle\nabla u \circ a \circ \nabla u\rangle d \tau \\
& +\int_{0}^{t} \frac{\sigma^{2}}{\mathbf{2}}\|f\|^{2} d \tau+\int_{0}^{t} \frac{\gamma^{2}}{\mathbf{2}}\left\|\mu_{3}\right\|^{2} d \tau .
\end{aligned}
$$

Assuming that $\varepsilon^{2}=\frac{\mathbf{1}}{\sqrt{\beta}}$ then $\frac{\mathbf{1}}{\mathbf{2}}\left(\frac{\mathbf{1}}{\varepsilon^{2}}+\beta \varepsilon^{2}\right)+\beta=\sqrt{\beta}+$ $\beta=\sqrt{\beta}(\mathbf{1}+\sqrt{\beta})$ and we are obtaining:

$$
\begin{aligned}
& \left.\frac{\mathbf{1}}{\mathbf{2}}\|u\|^{2}\right|_{0} ^{t}+\int_{0}^{t}\langle\nabla u \circ a \circ \nabla u\rangle d \tau+\lambda \int_{0}^{t}\|u\|^{2} d \tau \\
& \leq\left(\frac{\mathbf{1}}{\sqrt{\beta}}+\frac{c(\beta)}{\mathbf{2} \sqrt{\beta}}+c(\beta)\right) \int_{0}^{t}\|u\|^{2} d \tau \\
& +\sqrt{\beta}(\mathbf{1}+\sqrt{\beta}) \int_{0}^{t}\langle\nabla u \circ a \circ \nabla u\rangle d \tau \\
& +\frac{\sqrt{\beta}}{\mathbf{2}} \int_{0}^{t}\|f\|^{2} d \tau+\frac{\sqrt{\beta}}{\mathbf{2}} \int_{0}^{t}\left\|\mu_{3}\right\|^{2} d \tau .
\end{aligned}
$$

\section{The Smoothness of the Weak Solutions to the Quasilinear Second-Order Parabolic Partial Differential Equation (1)}

\section{Definition}

$A$ real-valued function $u(t, x) \in V_{\mathbf{1 , 0}}^{2}$ such that vrai $\max |u(t, x)|<\infty$ is called a weak bound solution to the quasilinear second-order parabolic partial differential Equation (1) if the identity:

$$
\begin{aligned}
& \left.\langle u(\tau), v(\tau)\rangle\right|_{0} ^{t}+\int_{0}^{t}\left(-\left\langle u(\tau), \partial_{t} v(\tau)\right\rangle+\lambda\langle u(\tau), v(\tau)\rangle\right) d \tau \\
& +\int_{0}^{t}\left\langle\sum_{i, j=\mathbf{1}, \ldots, l} a_{i j} \frac{\partial}{\partial x_{j}} u, \frac{\partial}{\partial x_{i}} v\right\rangle d \tau+\int_{0}^{t}\langle b, v\rangle d \tau=\int_{0}^{t}\langle f, v\rangle d \tau
\end{aligned}
$$

holds for all functions $v \in W_{10}^{2}$ such that vraimax $|v(t, x)|<\infty, t \in[\mathbf{0}, T]$.

For arbitrary function $v \in W_{\mathbf{1 , 0}}^{2}$ such that vraimax $|v(t, x)|<\infty, t \in[\mathbf{0}, T]$ from that definition of the weak solution we are obtaining 


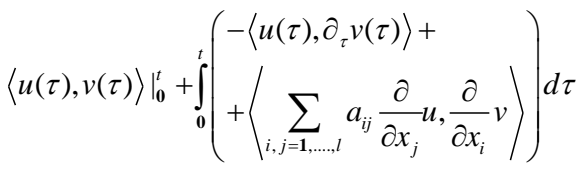

$$
\begin{aligned}
& \leq \int_{0}^{t}\langle f, v\rangle d \tau-\int_{0}^{t}(\lambda\langle u(\tau), v(\tau)\rangle) d \tau \\
& +\int_{0}^{t}\left\langle\mu_{1}(t, x)|\nabla u|+\mu_{2}(t, x)|u|+\mu_{3}(t, x), v(\tau)\right\rangle d \tau .
\end{aligned}
$$

Let $u(t, x)$ be a weak solution. We denote $v_{\bar{h}}(t, x)$ the average of function $v(t, x)$ at $\mathrm{t}$ by formulae:

$$
v_{\bar{h}}(t, x)=\frac{\mathbf{1}}{h} \int_{t-h}^{t} v(\tau, x) d \tau, u_{h}(t, x)=\frac{\mathbf{1}}{h} \int_{t}^{t+h} u(\tau, x) d \tau
$$

we transform:

$$
-\int_{0}^{T}\left\langle u \partial_{t} v_{\bar{h}}\right\rangle d t=-\int_{0}^{T}\left\langle u_{h} \partial_{t} v\right\rangle d t=\int_{0}^{T}\left\langle\partial_{t} u_{h} v\right\rangle d t
$$

since:

$$
\int_{0}^{T} u(t) v_{\bar{h}}(t) d t=\int_{0}^{T-h} u_{h}(t) v(t) d t
$$

where the function $v(t, x)$ is tautological equals zero over $t \leq 0$ and $T \geq t \geq T-h$.

\section{Remark}

The order of averaging and differentiation by $x$ are interchangeable.

Let us rewrite (6) as:

$$
\begin{aligned}
& \int_{0}^{T-h}\left(\left\langle\partial_{\tau} u_{h}, v\right\rangle+\lambda\left\langle u_{h}, v\right\rangle\right) d \tau \\
& +\int_{0}^{T-h}\left(\left\langle\left(\begin{array}{l}
\left.\sum_{i, j=1, \ldots, l} a_{i j} \frac{\partial}{\partial x_{j}} u\right), \\
+\left\langle b_{h}, v\right\rangle
\end{array}\right) \frac{\partial}{\partial x_{i}} v\right\rangle+\right) d \tau=\int_{0}^{T-h}\left\langle f_{h}, v\right\rangle d \tau
\end{aligned}
$$

Since in the last equality the function $v \in W_{1,0}^{2}$ is arbitrary, we can assume that $v=u_{h}$ next integrating with respect to $t$, we are passing to the limit as $h \rightarrow 0$ and are obtaining:

$$
\begin{aligned}
& \left.\frac{\mathbf{1}}{\mathbf{2}}\langle u, u\rangle\right|_{0} ^{t} \lambda \int_{0}^{t}\|u\|^{2} d \tau \\
& +\int_{0}^{t}\left(\left\langle\sum_{i, j=1, \ldots, l} a_{i j} \frac{\partial}{\partial x_{j}} u, \frac{\partial}{\partial x_{i}} u\right\rangle+\langle b, u\rangle\right) d \tau=\int_{0}^{t}\langle f, u\rangle d \tau .
\end{aligned}
$$

For an arbitrary function $v \in V_{1,0}^{2}$ the integrals:

$$
\int_{0}^{T-h}\left(\left\langle\left(\sum_{i, j=1, \ldots, l} a_{i j} \frac{\partial}{\partial x_{j}} u\right)_{h}, \frac{\partial}{\partial x_{i}} v\right\rangle+\left\langle b_{h}, v\right\rangle\right) d \tau
$$

and:

$$
\int_{0}^{T-h}\left\langle f_{h}, v\right\rangle d \tau
$$

converge to:

$$
\int_{0}^{T}\left(\left\langle\sum_{i, j=1, \ldots, l} a_{i j} \frac{\partial}{\partial x_{j}} u, \frac{\partial}{\partial x_{i}} v\right\rangle+\langle b, v\rangle\right) d \tau
$$

and:

$$
\int_{0}^{T}\langle f, v\rangle d \tau
$$

as $h \rightarrow 0$ so it is true for $v=u$.

For an arbitrary $t_{1}, t_{2} \in[h, T-h]$ applying (6) we can write:

$$
\begin{aligned}
& \int_{t_{1}}^{t_{2}}\left(\left\langle\partial_{\tau} u_{h}, v\right\rangle+\lambda\left\langle u_{h}, v\right\rangle\right) d \tau \\
& +\int_{t_{1}}^{t_{2}}\left(\left\langle\sum_{i, j=1, \ldots, l} a_{i j} \frac{\partial}{\partial x_{j}} u, \frac{\partial}{\partial x_{i}} v_{\bar{h}}\right\rangle+\left\langle b, v_{\bar{h}}\right\rangle\right) d \tau=\int_{t_{1}}^{t_{2}}\left\langle f_{h}, v\right\rangle d \tau,
\end{aligned}
$$

assume $v=u_{h}^{k}$, where $u^{k}(t, x) \equiv \max [u(t, x)-k, 0]$ and we denote the set of points $P_{k}(t)=\left\{x \in R^{l}: u(t, x)>k, t \in[0\right.$, $T]\}, R^{l}, l>2$ and $P_{k}(t)=\left\{(t, x) \in[0, \tau] \times R^{l}: u(\tau, x)>k\right.$, $\tau \in[0, T], l>2\}$, we have:

$$
\begin{aligned}
& \frac{\mathbf{1}}{\mathbf{2}}\left\|u^{k}(t)\right\|_{P_{k}(t)}{ }^{2}+\int_{0}^{t}\left\langle\nabla u^{k} \circ a \circ \nabla u^{k}\right\rangle_{P_{k}(t)} d \tau \\
& +\lambda \int_{0}^{t}\left\|u^{k}\right\|_{P_{k}(t)}^{2} d \tau \\
& \leq\left(\frac{\mathbf{1}}{\sqrt{\beta}}+\frac{c(\beta)}{\mathbf{2} \sqrt{\beta}}+c(\beta)\right) \int_{0}^{t}\|u\|_{P_{k}(t)}^{2} d \tau \\
& +\sqrt{\beta}(\mathbf{1}+\sqrt{\beta}) \int_{\mathbf{0}}^{t}\langle\nabla u \circ a \circ \nabla u\rangle_{P_{k}(t)} d \tau \\
& +\frac{\sqrt{\beta}}{\mathbf{2}} \int_{\mathbf{0}}^{t}\|f\|_{P_{k}(t)}^{2} d \tau+\frac{\sqrt{\beta}}{\mathbf{2}} \int_{0}^{t}\left\|\mu_{3}\right\|_{P_{k}(t)}{ }^{2} d \tau .
\end{aligned}
$$

From $(a+b)^{2} \leq \mathbf{2}\left(a^{2}+b^{2}\right)$, we obtain: 


$$
\int_{0}^{t}\|u\|_{P_{k}(t)}^{2} d \tau \leq \mathbf{2}\left(\|u-k\|_{P_{k}(t)}{ }^{2}+k^{2} \int_{0}^{t} m e s P_{k}(\tau) d \tau\right) .
$$

\section{Lemma 1}

Let element $u \in V_{0}^{2}$ satisfies following tautology:

$$
\begin{aligned}
& \int_{0}^{T}\left(-\left\langle u, \partial_{\tau} \varphi\right\rangle+\lambda\langle u, \varphi\rangle\right) d \tau \\
& +\int_{0}^{T}\left(\left\langle\sum_{i, j=1, \ldots, l} a_{i j} \frac{\partial}{\partial x_{j}} u, \frac{\partial}{\partial x_{i}} \varphi\right\rangle+\langle b, \varphi\rangle\right) d \tau \\
& =\int_{0}^{T}\langle f, \varphi\rangle d \tau, f \in L^{2}
\end{aligned}
$$

where the $\varphi$ is an arbitrary element of functional space $W_{1,0}^{2}\left([\mathbf{0}, T] \times R^{l}\right)$ then element $u \in V_{0}^{2}$ belongs $V_{1, \frac{1}{2}}^{2}\left([0, T] \times R^{l}\right)$.

Space $V_{\mathbf{1}, \frac{1}{2}}^{2}\left([\mathbf{0}, T] \times R^{l}\right)$ is a subspace of $W_{\mathbf{1}, \mathbf{0}}^{2}\left([\mathbf{0}, T] \times R^{l}\right)$ that consists of all continuous at $t$ in $L^{2}\left(R^{l}\right)$ norm elements with the norm $\|u\|_{V}=\max _{t \in[0, T]}\|u(t)\|+\left\|\nabla_{i} u\right\|_{[0, T] \times R^{\prime}}$ and the following condition $\int_{0}^{T-p}\left\langle\frac{\mathbf{1}}{h}|u(t+h, \cdot)-u(t, \cdot)|^{2}\right\rangle d t \stackrel{h \rightarrow 0}{\longrightarrow} \mathbf{0}$ is satisfied.

\section{Proof of Lemma 1}

For arbitrary $\varphi \in W_{\mathbf{1 , 0}}^{2}\left([\mathbf{0}, T] \times R^{l}\right) \quad$ we denote $\varphi_{\bar{h}}(t, x)=\frac{\mathbf{1}}{h} \int_{t-h}^{t} \varphi(\tau, x) d \tau$ then:

$$
\begin{aligned}
& \int_{0}^{T}\left(-\left\langle u_{h}, \partial_{\tau} \varphi\right\rangle+\lambda\left\langle u_{h}, \varphi\right\rangle\right) d \tau \\
& +\int_{0}^{T}\left(\left\langle\left(\sum_{i, j=1, \ldots, l} a_{i j} \frac{\partial}{\partial x_{j}} u\right)_{h}, \frac{\partial}{\partial x_{i}} \varphi\right\rangle+\left\langle b_{h}, \varphi\right\rangle\right) d \tau \\
& =\int_{0}^{T}\left\langle f_{h}, \varphi\right\rangle d \tau,
\end{aligned}
$$

put $\varphi(t, x)=\chi(t) \psi(x)$, where $\chi(t)$ is a smooth function of time and $\psi \in W_{1,0}^{2}\left(R^{l}\right)$. We have:

$$
\begin{aligned}
& \int_{-\infty}^{\infty}\left(-\partial_{\tau} \chi(\tau)\left\langle u_{h}, \psi\right\rangle+\lambda \chi(\tau)\left\langle u_{h}, \psi\right\rangle\right) d \tau \\
& +\int_{-\infty}^{\infty} \chi(\tau)\left(\left\langle\left(\sum_{i, j=1, \ldots, l} a_{i j} \frac{\partial}{\partial x_{j}} u\right)_{h}, \frac{\partial}{\partial x_{i}} \psi\right\rangle+\left\langle b_{h}, \psi\right\rangle\right) d \tau \\
& =\int_{-\infty}^{\infty} \chi(\tau)\left\langle f_{h}, \psi\right\rangle d \tau,
\end{aligned}
$$

so:

$$
\begin{aligned}
& \partial_{\tau}\left\langle u_{h}, \psi\right\rangle+\lambda\left\langle u_{h}, \psi\right\rangle+\left\langle\left(\sum_{i, j=1, \ldots, l} a_{i j} \frac{\partial}{\partial x_{j}} u\right)_{h}, \frac{\partial}{\partial x_{i}} \psi\right\rangle \\
& +\left\langle b_{h}, \psi\right\rangle=\left\langle f_{h}, \psi\right\rangle \forall \psi \in W_{\mathbf{1}, \mathbf{0}}^{2}\left(R^{l}\right),
\end{aligned}
$$

and:

$$
\begin{aligned}
& \left\langle\partial_{\tau} u_{h}, \psi\right\rangle+\lambda\left\langle u_{h}, \psi\right\rangle+\left\langle\left(\sum_{i, j=1, \ldots, l} a_{i j} \frac{\partial}{\partial x_{j}} u\right)_{h}, \frac{\partial}{\partial x_{i}} \psi\right\rangle \\
& +\left\langle b_{h}, \psi\right\rangle=\left\langle f_{h}, \psi\right\rangle \forall \psi \in W_{\mathbf{1}, \mathbf{0}}^{2}\left(R^{l}\right),
\end{aligned}
$$

and for arbitrary $h_{1}, h_{2}$, we have:

$$
\begin{aligned}
& \left\langle\partial_{\tau} u_{h_{1}}-\partial_{\tau} u_{h_{2}}, \psi\right\rangle+\lambda\left\langle u_{h_{1}}-u_{h_{2}}, \psi\right\rangle \\
& +\left\langle\left(\sum_{i, j=1, \ldots, l} a_{i j} \frac{\partial}{\partial x_{j}} u\right)_{h_{1}}-\left(\sum_{i, j=1, \ldots, l} a_{i j} \frac{\partial}{\partial x_{j}} u\right)_{h_{2}}, \frac{\partial}{\partial x_{i}} \psi\right\rangle \\
& +\left\langle b_{h_{1}}-b_{h_{2}}, \psi\right\rangle=\left\langle f_{h_{1}}-f_{h_{2}}, \psi\right\rangle \forall \psi \in W_{1,0}^{2}\left(R^{l}\right),
\end{aligned}
$$

assuming that $\psi=u_{h_{1}}-u_{h_{2}}$ then we are obtaining:

$$
\begin{aligned}
& \frac{\mathbf{1}}{\mathbf{2}} \partial_{\tau}\left\|u_{h_{1}}-u_{h_{2}}\right\|^{2}+\lambda\left\|u_{h_{1}}-u_{h_{2}}\right\|^{2} \\
& +\left(\begin{array}{l}
\left.\sum_{i, j=1, \ldots, l} a_{i j} \frac{\partial}{\partial x_{j}} u\right)_{h_{1}}- \\
-\left(\sum_{i, j=1, \ldots, l} a_{i j} \frac{\partial}{\partial x_{j}} u\right)_{h_{2}}, \frac{\partial}{\partial x_{i}}\left(u_{h_{1}}-u_{h_{2}}\right)
\end{array}\right) \\
& +\left\langle b_{h_{1}}-b_{h_{2}}, u_{h_{1}}-u_{h_{2}}\right\rangle=\left\langle f_{h_{1}}-f_{h_{2}}, u_{h_{1}}-u_{h_{2}}\right\rangle,
\end{aligned}
$$

by integrating with respect to time, we have:

$$
\begin{aligned}
& \left.\frac{\mathbf{1}}{\mathbf{2}}\left\|u_{h_{1}}-u_{h_{2}}\right\|^{2}\right|_{t_{1}} ^{t_{2}}+\lambda \int_{t_{1}}^{t_{2}}\left\|u_{h_{1}}-u_{h_{2}}\right\|^{2} d \tau \\
& +\int_{t_{1}}^{t_{2}}\left\langle\begin{array}{l}
\left.\sum_{i, j=1, \ldots, l} a_{i j} \frac{\partial}{\partial x_{j}} u\right)_{h_{1}}- \\
-\left(\sum_{i, j=1, \ldots, l} a_{i j} \frac{\partial}{\partial x_{j}} u\right)_{h_{2}}, \frac{\partial}{\partial x_{i}}\left(u_{h_{1}}-u_{h_{2}}\right)
\end{array}\right) d \tau \\
& +\int_{t_{1}}^{t_{2}}\left\langle b_{h_{1}}-b_{h_{2}}, u_{h_{1}}-u_{h_{2}}\right\rangle d \tau \\
& =\int_{t_{1}}^{t_{2}}\left\langle f_{h_{1}}-f_{h_{2}}, u_{h_{1}}-u_{h_{2}}\right\rangle d \tau, \quad t_{1}, t_{2} \in[\mathbf{0}, T] .
\end{aligned}
$$

Let pass to limit as $h_{\mathbf{1}} \rightarrow \mathbf{0}, h_{2} \rightarrow \mathbf{0}$ we obtain: 


$$
\begin{aligned}
& \left\|u_{h_{1}}-u_{h_{2}}\right\|+\left\|\frac{\partial}{\partial x_{i}}\left(u_{h_{1}}-u_{h_{2}}\right)\right\| \\
& +\left\|\left(\sum_{i, j=1, \ldots, l} a_{i j} \frac{\partial}{\partial x_{j}} u\right)_{h_{1}}-\left(\sum_{i, j=1, \ldots, l} a_{i j} \frac{\partial}{\partial x_{j}} u\right)_{h_{2}}\right\| \\
& +\left\|b_{h_{1}}-b_{h_{2}}\right\|+\left\|f_{h_{1}}-f_{h_{2}}\right\| \stackrel{h_{1}, h_{2} \rightarrow 0}{\rightarrow} \mathbf{0} .
\end{aligned}
$$

We denote $\psi(x)=\Delta_{h} u \equiv u(t+h, x)-u(t, x)$ then:

$$
\begin{aligned}
& \int_{-\infty}^{\infty}\left(\begin{array}{l}
\left\langle\partial_{\tau} u_{h}, u(t+h, x)-u(t, x)\right\rangle+ \\
+\lambda\left\langle u_{h}, u(t+h, x)-u(t, x)\right\rangle
\end{array}\right) d t \\
& +\int_{-\infty}^{\infty}\left\langle\left(\sum_{i, j=1, \ldots, l} a_{i j} \frac{\partial}{\partial x_{j}} u\right)_{h}, \frac{\partial}{\partial x_{i}}(u(t+h, x)-u(t, x))\right\rangle d t \\
& +\int_{-\infty}^{\infty}\left\langle b_{h}, u(t+h, x)-u(t, x)\right\rangle d t \\
& =\int_{-\infty}^{\infty}\left\langle f_{h}, u(t+h, x)-u(t, x)\right\rangle d t
\end{aligned}
$$

and we have:

$$
\begin{aligned}
& \int_{-\infty}^{\infty} \frac{\left\|\Delta_{h} u\right\|^{2}}{h} d t+\lambda \int_{-\infty}^{\infty}\left\langle u_{h}, \Delta_{h} u\right\rangle d t \\
& +\int_{-\infty}^{\infty}\left\langle\Delta_{h}\left(\sum_{i, j=1, \ldots, l} a_{i j} \frac{\partial}{\partial x_{j}} u\right), \frac{\partial}{\partial x_{i}} u_{h}\right\rangle d t+\int_{-\infty}^{\infty}\left\langle\Delta_{h} b, u_{h}\right\rangle d t \\
& =\int_{-\infty}^{\infty}\left\langle\Delta_{h} f, u_{h}\right\rangle d t,
\end{aligned}
$$

Applying Holder inequality and previous considerations, we have obtained:

$$
\int_{-\infty}^{\infty} \frac{\left\|\Delta_{h} u\right\|_{L^{2}\left(R^{l}\right)}^{2}}{h} d t \leq \varepsilon(h) \stackrel{h \rightarrow 0}{\longrightarrow} \mathbf{0},
$$

that proves the lemma.

\section{A Priori Estimation of the Solution to (1)}

Let us assume that ellipticity condition and (4), (5) are satisfied and all weak solutions $u(t, x)$ of the $V_{1,0}^{2}$ are bounded, we will show that $u \in H^{\alpha, \frac{\alpha}{2}}$ for certain $\alpha>0$ and estimate the norm $|u|^{(\alpha)}$.

Assume $u \in V_{1,0}^{2}$ for arbitrary element $\varphi \in W_{1,0}^{2}$, we have tautology (6) and we obtain an estimation:

$$
\begin{aligned}
& \left.\langle u(\tau), \varphi(\tau)\rangle\right|_{t_{1}} ^{t_{2}} \\
& +\int_{t_{1}}^{t_{2}}\left(-\left\langle u(\tau), \partial_{\tau} \varphi(\tau)\right\rangle+\lambda\langle u(\tau), \varphi(\tau)\rangle\right) d \tau
\end{aligned}
$$

$$
\begin{aligned}
& +\int_{t_{1}}^{t_{2}}\left\langle\sum_{i, j=1, \ldots, l} a_{i j} \frac{\partial}{\partial x_{j}} u, \frac{\partial}{\partial x_{i}} \varphi\right\rangle d \tau \\
& \leq\left|\int_{t_{1}}^{t_{2}}\langle b, \varphi\rangle d \tau\right|+\int_{t_{1}}^{t_{2}}|\langle f, \varphi\rangle| d \tau,
\end{aligned}
$$

since for arbitrary element $\varphi \in W_{1,0}^{2}$, the following condition is executed:

$$
\left|\int_{t_{1}}^{t_{2}}\langle b, \varphi\rangle d \tau\right| \leq\left|\int_{t_{1}}^{t_{2}}\left\langle\mu_{1}|\nabla u|+\mu_{2}|u|+\mu_{3},|\varphi|\right\rangle d \tau\right|,
$$

so:

$$
\begin{aligned}
& \left.\langle u(\tau), \varphi(\tau)\rangle\right|_{t_{1}} ^{t_{1}}+\int_{t_{1}}^{t_{2}}\left(-\left\langle u(\tau), \partial_{\tau} \varphi(\tau)\right\rangle+\lambda\langle u(\tau), \varphi(\tau)\rangle\right) d \tau \\
& +\int_{t_{1}}^{t_{2}}\left\langle\sum_{i, j=1, \ldots, l} a_{i j} \frac{\partial}{\partial x_{j}} u, \frac{\partial}{\partial x_{i}} \varphi\right\rangle d \tau \\
& \leq \int_{t_{1}}^{t_{2}}\left(\left\langle\mu_{1}|\nabla u|,|\varphi|\right\rangle+\left\langle\mu_{2}|u|,|\varphi|\right\rangle+\left\langle\mu_{3},|\varphi|\right\rangle\right) d \tau \\
& +\int_{t_{1}}^{t_{2}}|\langle f, \varphi\rangle| d \tau,
\end{aligned}
$$

let us put $\varphi(t, x)=(\xi(t, x))^{2} u(t, x) \equiv \xi^{2} u, \quad$ and integrate by parts, we are obtaining

$$
\begin{aligned}
& \frac{\mathbf{1}}{\mathbf{2}}\|u(\tau) \xi(\tau)\|_{K(\delta)}^{2} l_{t_{1}}^{t_{2}}+ \\
& +\int_{t_{1}}^{t_{2}}\left(\begin{array}{l}
\left.-\left\langle u^{2}(\tau) \xi(\tau), \partial_{\tau} \xi(\tau)\right\rangle_{K(\delta)}+\right)^{2}+\lambda\left\langle u^{2}(\tau), \xi^{2}(\tau)\right\rangle_{K(\delta)}
\end{array}\right) d \tau \\
& +\int_{t_{1}}^{t_{2}}\left(\sum_{i, j=1, \ldots, l} a_{i j} \frac{\partial}{\partial x_{j}} u, \frac{\partial}{\partial x_{i}}\left(u \xi^{2}(\tau)\right)\right\rangle_{K(\delta)} d \tau \\
& \leq \int_{t_{1}}^{t_{2}}\left(\begin{array}{l}
\left\langle\mu_{1}|\nabla u|,\left|u \xi^{2}(\tau)\right|\right\rangle_{K(\delta)}+ \\
+\left\langle\mu_{2}|u|,\left|u \xi^{2}(\tau)\right|\right\rangle_{K(\delta)}+ \\
+\left\langle\mu_{3},\left|u \xi^{2}(\tau)\right|\right\rangle_{K(\delta)}
\end{array}\right) d \tau \\
& +\int_{t_{1}}^{t_{2}}\left\langle\left\langle f, u \xi^{2}(\tau)\right\rangle_{K(\delta)}\right| d \tau,
\end{aligned}
$$

where the $K(\delta)$ is a cube in $R^{l}$ with an edge length of $\delta$.

Next, we estimate:

$$
\begin{aligned}
& \left|\left\langle\mu_{1}|\nabla u|,|u| \xi^{2}(\tau)\right\rangle\right| \leq\left\|\mu_{1} \xi^{2}(\tau)\right\|\||\nabla u| u\| \\
& \leq \frac{\mathbf{1}}{\mathbf{2}}\left(\frac{\mathbf{1}}{\varepsilon^{2}}\left\|\mu_{1} \xi^{2}(\tau)\right\|^{2}+\varepsilon^{2}\|\nabla u \mid u\|^{2}\right),
\end{aligned}
$$




$$
\begin{aligned}
& \left\|\mu_{1} \xi^{2}(\tau)\right\|^{2}=\left\langle\left(\mu_{1} \xi^{2}(\tau)\right)^{2}\right\rangle \\
& \leq \beta\left\langle\nabla \xi^{2} \circ a \circ \nabla \xi^{2}\right\rangle+c(\beta)\left\|\xi^{2}\right\|^{2},
\end{aligned}
$$

similarly:

$$
\begin{aligned}
& \left\langle\mu_{3} \xi^{2}(\tau), u\right\rangle \leq\left\|\mu_{3} \xi^{2}(\tau)\right\|\|u\| \leq \\
& \leq\left(\beta\langle\nabla \xi \circ a \circ \nabla \xi\rangle+c(\beta)\|\xi\|^{2}\right)^{\frac{1}{2}}\|u\|
\end{aligned}
$$

and:

$$
\|\nabla u \mid u\|^{2} \leq \frac{\mathbf{1}}{\mathbf{2}}\left(\frac{\mathbf{1}}{\varepsilon_{1}^{2}}\left\|\left.\nabla u\right|^{2}\right\|^{2}+\varepsilon_{1}^{2}\left\|u^{2}\right\|^{2}\right) .
$$

These we have had the following inequality:

$$
\begin{aligned}
& \left\|u\left(t_{2}\right) \xi\left(t_{2}\right)\right\|_{K(\delta)}^{2} \\
& +K \int_{t_{1}}^{t_{2}}\left\langle\sum_{i, j=1, \ldots, l} a_{i j} \frac{\partial}{\partial x_{j}} u, \frac{\partial}{\partial x_{i}}\left(u \xi^{2}(\tau)\right)\right\rangle_{K(\delta)} d \tau \\
& \leq\left\|u\left(t_{1}\right) \xi\left(t_{1}\right)\right\|_{K(\delta)}^{2} \\
& +\int_{t_{1}}^{t_{2}}\left(K_{1}\|\nabla \xi\|+K_{2}\|\xi\|+K_{3}\left\langle\xi\left|\xi_{\tau}\right|\right\rangle\right)_{K(\delta)} d \tau \\
& +K_{4}^{t_{2}} \int_{t_{1}}^{t_{1}}\left(F\left(f, \xi^{2}\right)\|u\|\right)_{K(\delta)} d \tau,
\end{aligned}
$$

where $K, K_{1}, K_{2}, K_{3}$ are positive constants depended on the initial conditions and constants $\varepsilon, \varepsilon_{1}, \ldots, \varepsilon_{4}$ are arbitrary constants, such that:

$$
\begin{aligned}
& \frac{\mathbf{1}}{\mathbf{2}}\left(\frac{\mathbf{1}}{\varepsilon^{2}}\left\|\mu_{1} \xi^{2}(\tau)\right\|^{2}+\varepsilon^{2}\|\nabla u \mid u\|^{2}\right) \\
& \leq \frac{\mathbf{1}}{\mathbf{2}}\left(\begin{array}{l}
\frac{\mathbf{1}}{\varepsilon^{2}} \beta\left\langle\nabla \xi^{2} \circ a \circ \nabla \xi^{2}\right\rangle+c(\beta)\left\|\xi^{2}\right\|^{2}+ \\
+\varepsilon^{2} \frac{\mathbf{1}}{\mathbf{2}}\left(\frac{\mathbf{1}}{\varepsilon_{1}^{2}}\left\|\left.\nabla u\right|^{2}\right\|^{2}+\varepsilon_{1}^{2}\left\|u^{2}\right\|^{2}\right)
\end{array}\right),
\end{aligned}
$$

it is possible to presume $\varepsilon^{2}=c \beta$, where $c$ is a constant. Thus we have obtained a prior estimation for the solution to the equation (1).

Let us assume the function $u \in V_{1,0}^{2}$ is a solution to the equation (1) then for an arbitrary element $v \in W_{1,0}^{2}\left(R^{l}, d^{l} x\right)$ such that vraimax $|v(t, x)|<\infty, t \in[\mathbf{0}, T]$, we have an integral equality:

$$
\begin{aligned}
& \left.\langle u(\tau), v(\tau)\rangle\right|_{0} ^{t}+\int_{0}^{t}\left(-\left\langle u(\tau), \partial_{t} v(\tau)\right\rangle+\lambda\langle u(\tau), v(\tau)\rangle\right) d \tau \\
& +\int_{0}^{t}\left\langle\left(\frac{\partial}{\partial x_{i}} \sum_{i, j=1, \ldots, l} a_{i j}\right) \frac{\partial}{\partial x_{j}} u, v\right\rangle d \tau \\
& +\int_{0}^{t}\left\langle\sum_{i, j=1, \ldots, l} a_{i j}\left(\frac{\partial}{\partial x_{i}} \frac{\partial}{\partial x_{j}} u\right), v\right\rangle d \tau \\
& +\int_{0}^{t}\langle b, v\rangle d \tau=\int_{0}^{t}\langle f, v\rangle d \tau .
\end{aligned}
$$

We put $v=u$ and obtain:

$$
\begin{aligned}
& \left.\frac{\mathbf{1}}{\mathbf{2}}\|u(\tau)\|^{2}\right|_{0} ^{t}+\lambda \int_{0}^{t}\|u(\tau)\|^{2} d \tau \\
& +\int_{0}^{t}\left\langle\left(\frac{\partial}{\partial x_{i}} \sum_{i, j=1, \ldots, l} a_{i j}\right) \frac{\partial}{\partial x_{j}} u, u\right\rangle d \tau \\
& +\int_{0}^{t}\left\langle\sum_{i, j=1, \ldots, l} a_{i j}\left(\frac{\partial}{\partial x_{i}} \frac{\partial}{\partial x_{j}} u\right), u\right\rangle d \tau \\
& =\int_{0}^{t}\langle f, u\rangle d \tau-\int_{0}^{t}\langle b, u\rangle d \tau .
\end{aligned}
$$

The right part can be estimated similarly to previous considerations with an application of Holder and Young inequalities.

The elliptic condition can be presented as:

$$
v\|\xi\|^{2} \leq \sum_{i j=1, \ldots, l} a_{i j} \xi_{i} \xi_{j} \leq \mu\|\xi\|^{2} \quad \forall \xi \in R^{l}
$$

so form $B(\xi, v) \equiv \sum_{i j=1, \ldots, l} a_{i j} \xi_{i} v_{j}$ defines a certain metric and $\sum_{i j=1 \ldots, l} a_{i j} \xi_{i} v_{j} \leq\|\xi\|_{B}\|\nu\|_{B}$, where the norm $\|\cdot\|_{B}$ is generated by the form $B$. Then there is a constant $\sqrt{\gamma}$ such that $\|\xi\|_{B} \leq \sqrt{\gamma}\|\xi\|$ so the estimation $\sum_{i j=1, \ldots, l} a_{i j} \xi_{i} v_{j} \leq \gamma\|\xi\|\|v\|$ is true.

Thus, we have obtained that there is a constant $C_{1}$ such that:

$\left\langle\sum_{i, j=1, \ldots, l} a_{i j}\left(\frac{\partial}{\partial x_{i}} \frac{\partial}{\partial x_{j}} u\right), u\right\rangle \leq C_{1}\|\Delta u\|\|u\|$.

\section{Theorem 1}

Assuming that the Cauchy's problem:

$$
\begin{gathered}
\frac{\partial}{\partial t} u+\lambda u-\frac{\partial}{\partial x_{i}}\left(a_{i j}(t, x, u) \frac{\partial}{\partial x_{j}} u\right)+b^{k}(t, x, u, \nabla u)=f(t, x), \\
u(\mathbf{0}, x)=u_{0}(x),
\end{gathered}
$$


under the form-bounded of $b$ and vraimax $\left|\frac{\partial a_{j k}}{\partial x_{i}}\right|<\infty$ conditions has a solution $u \in W_{1,1}^{2}$, then the solution belongs $W_{2,1}^{2}$.

\section{The Existence of the Solution to the}

\section{Parabolic Partial Differential Equation (1)}

Theorem 2

The quasi-linear parabolic partial differential Equation (1) under the conditions (4), (5) has the solution from $W_{\mathbf{1}}^{2}\left([\mathbf{0}, T] \times R^{l}\right)$.

\section{Proof}

To prove the existence of the solution to (1) we construct the sequence of approximate solutions $\left\{u_{m}(t, x)\right\}, m=\mathbf{1}, \mathbf{2}, \ldots$ to the equation:

$$
\frac{\partial}{\partial t} u+\lambda u-\frac{\partial}{\partial x_{i}}\left(a_{i j}(t, x, u) \frac{\partial}{\partial x_{j}} u\right)+b(t, x, u, \nabla u)=f,
$$

as $\left\{u_{m}(t, x)\right\}=\left\{\sum_{i=1}^{m} c_{i}^{m}(t) \varphi_{i}(x)\right\}$, where the elements $\left\{\varphi_{n}(x)\right\}$ $n=\mathbf{1}, \mathbf{2}, \ldots$. form the basis of $W_{1}^{2}\left(R^{l}\right)$ with the properties $\left(\varphi_{i}, \varphi_{j}\right)=\delta_{i j}$ and $\max _{R^{I}}\left|\varphi_{i}, \varphi_{i x}\right| \leq c_{i}<\infty$. The functional coefficients $c_{n}^{m}(t)$ of $\left\{u_{m}(t, x)\right\}==\left\{\sum_{i=1}^{m} c_{i}^{m}(t) \varphi_{i}(x)\right\} \quad$ are determined by:

$$
\begin{aligned}
& \left\langle\partial_{t} u_{m}, \varphi_{n}\right\rangle+\lambda\left\langle u_{m}, \varphi_{n}\right\rangle \\
& +\left\langle\sum_{i, j=\mathbf{1}, \ldots, l} a_{i j} \frac{\partial}{\partial x_{j}} u_{m}, \frac{\partial}{\partial x_{i}} \varphi_{n}\right\rangle+\left\langle b, \varphi_{n}\right\rangle=\left\langle f, \varphi_{n}\right\rangle, n=\mathbf{1}, \mathbf{2}, \ldots, m
\end{aligned}
$$

and initial conditions:

$$
c_{n}^{m}(\mathbf{0})=\left(u_{\mathbf{0}}, \varphi_{n}(x)\right), n=\mathbf{1}, \mathbf{2}, \ldots, m .
$$

From the initial conditions for $t \in[0, T]$ we are obtaining $\quad\left|c_{n}^{m}\right| \leq$ const $, n=\mathbf{1}, \mathbf{2}, \ldots, m$, from ellipticity follows uniformly boundedness of the solutions over $t \in$ $[0, T]$, to show this we multiply the Equation (1) by $c_{n}^{m}$ and a sum of $n$ up to $m$ then we obtain the inequality:

$$
\begin{aligned}
& \frac{\mathbf{1}}{\mathbf{2}}\left\|u_{m}(t)\right\|^{2}+\int_{\mathbf{0}}^{t}\left\langle\nabla u_{m} \circ a \circ \nabla u_{m}\right\rangle d \tau+\lambda \int_{0}^{t}\left\|u_{m}\right\|^{2} d \tau \\
& \leq\left(\frac{\mathbf{1}}{\sqrt{\beta}}+\frac{c(\beta)}{\mathbf{2} \sqrt{\beta}}+c(\beta)\right) \int_{0}^{t}\left\|u_{m}\right\|^{2} d \tau
\end{aligned}
$$

$$
\begin{aligned}
& +\sqrt{\beta}(\mathbf{1}+\sqrt{\beta}) \int_{0}^{t}\left\langle\nabla u_{m} \circ a \circ \nabla u_{m}\right\rangle d \tau \\
& +\frac{\sqrt{\beta}}{\mathbf{2}} \int_{\mathbf{0}}^{t}\|f\|^{2} d \tau+\frac{\sqrt{\beta}}{\mathbf{2}} \int_{0}^{t}\left\|\mu_{3}\right\|^{2} d \tau .
\end{aligned}
$$

We will apply the following lemma.

\section{Lemma 2}

Let $\psi(t)$ be a positive absolute continuous function such that $\psi(\mathbf{0})=\mathbf{0}$ and for almost all $t \in[\mathbf{0}, T]$ holds the inequality:

$\frac{d}{d} \psi(t) \leq c(t) \psi(t)+F(t)$

where the $c(t)$ and $F(t)$ are positive integratable on $[\mathbf{0 , T}]$ functions. Then:

$\psi(t) \leq \exp \left(\int_{0}^{t} c(\tau) d \tau\right) \int_{0}^{t} F(\tau) d \tau$

and:

$\frac{d}{d} \psi(t) \leq c(t) \exp \left(\int_{0}^{t} c(\tau) d \tau\right) \int_{0}^{t} F(\tau) d \tau+F(t)$.

Since $u_{0} \in L^{2}\left(R^{l}\right)$ there is an estimation:

$$
\max _{t \in[0, T]} \sum_{n=1}^{m}\left(c_{n}^{m}(t)\right)^{2}=\max _{t \in[0, T]}\left\|u_{m}\right\|^{2} \leq \text { const }
$$

Functions $\quad c_{n}^{m}(t)=\left(u^{m}(t, x), \varphi_{n}(x)\right), m, n=\mathbf{1}, \mathbf{2}, \ldots . \quad$ are continuous on $[\mathbf{0}, T]$. On the interval $[t, t+\Delta t]$, we can estimate:

$$
\begin{aligned}
& \left\langle\left|u_{m}(t+\Delta t, x)-u_{m}(t, x)\right|, \varphi_{n}\right\rangle \\
& \leq \int_{t}^{t+\Delta t}\left(\left\langle\left|\sum_{i, j=1, \ldots, l} a_{i j} \frac{\partial}{\partial x_{j}} u_{m}, \frac{\partial}{\partial x_{i}} \varphi_{n}\right|\right\rangle d \tau\right. \\
& +\int_{t}^{t+\Delta t}\left\langle f, \varphi_{n}\right\rangle d \tau+\lambda \int_{t}^{t+\Delta t}\left\langle u_{m}, \varphi_{n}\right\rangle d \tau \\
& +\int_{t}^{t+\Delta t}\left\langle\mu_{1}(t, x)\left|\nabla u_{m}\right|+\mu_{2}(t, x)\left|u_{m}\right|+\right\rangle d \tau \\
& \leq c_{n} \int_{t}^{t+\Delta t}\left(\left\langle\mu_{3}(t, x), \varphi_{n}\right.\right. \\
& -\lambda c_{n} \int_{t}^{t+\Delta t} \mid\left\|u_{m}\right\| d \tau
\end{aligned}
$$




$$
\begin{aligned}
& +c_{n} \operatorname{const}(\beta) \int_{t}^{t+\Delta t}\left\|u_{m}\right\|^{2} d \tau \\
& +c_{n} \operatorname{const}(\beta) \int_{t}^{t+\Delta t}\left\langle\nabla u_{m} \circ a \circ \nabla u_{m}\right\rangle d \tau \\
& +c_{n} \operatorname{const}(\beta)\left(\int_{t}^{t+\Delta t}\|f\|^{2} d \tau+\int_{t}^{t+\Delta t}\left\|\mu_{3}\right\|^{2} d \tau\right) \\
& \leq \operatorname{Const}(n, \varphi, l) \Delta t .
\end{aligned}
$$

Thus, constants $\operatorname{Const}(n, \varphi, l)$ depend on $n, \varphi, l$ but do not depend on $m$ under the condition $m \geq n$ so:

$$
\left|c_{n}^{m}(t+\Delta t)-c_{n}^{m}(t)\right| \leq \varepsilon(\Delta t)\left\|\varphi_{n}\right\| \underset{t \rightarrow \mathbf{0}}{\rightarrow \mathbf{0}}
$$

Applying the diagonal method we are obtaining that the sequence $c_{n}^{m(i)}, i=\mathbf{1}, \mathbf{2}, \ldots$. converges uniformly on $[0, T]$ to a certain continuous function $c_{n}(t), n=\mathbf{1}, \mathbf{2}, \ldots$ for every $n$. The sequence of functions $c_{n}(t), n=\mathbf{1}, \mathbf{2}, \ldots$. determines the function $u(t, x)$ as a $L^{2}\left(R^{l}\right)$-weak uniformly on $[0, T]$ limit of the functional sequence $\left\{u_{m}(t, x)\right\}=\left\{\sum_{i=1}^{m} c_{i}^{m}(t) \varphi_{i}(x)\right\} \quad$ that converges to $u(t, x)=\sum_{i=1}^{\infty} c_{i}(t) \varphi_{i}(x)$. To show the weak convergence we consider the equality:

$$
\begin{aligned}
& \left(u_{m(i)}-u, v\right)=\sum_{n=1}^{s}\left(v, \varphi_{n}\right)\left(u_{m(i)}-u, \varphi_{n}\right) \\
& +\left(u_{m(i)}-u, \sum_{n=s+1}^{\infty}\left(v, \varphi_{n}\right) \varphi_{n}\right),
\end{aligned}
$$

and apply estimation:

$$
\left|\left(u_{m(i)}-u, \sum_{n=s+1}^{\infty}\left(v, \varphi_{n}\right) \varphi_{n}\right)\right| \leq \mathrm{const}\left(\sum_{n=s+1}^{\infty}\left(v, \varphi_{n}\right)^{2}\right)^{\frac{1}{2}} .
$$

Let $s$ be large enough number so for any fixed real number $\varepsilon$ there is inequality:

$$
\operatorname{const}\left(\sum_{n=s+1}^{\infty}\left(v, \varphi_{n}\right)^{2}\right)^{\frac{1}{2}} \leq \frac{\varepsilon}{2}
$$

and for large enough $m(i)$ the first sum also less that $\frac{\varepsilon}{2}$ for all $t \in[0, T]$.

Let us show that the function $u$ is a solution to the Cauchy problem for (1). For arbitrary function $v=\sum_{i=1}^{m} d_{i}^{m}(t) \varphi_{i}(x)$, where the $d_{i}^{m}(t)$ are arbitrary continuous functions with bounded weak derivatives, we consider the equality;

$$
\begin{aligned}
& \left.\left\langle u_{m}(\tau), v(\tau)\right\rangle\right|_{0} ^{t}+\int_{0}^{t}\left(-\left\langle u_{m}(\tau), \partial_{t} v(\tau)\right\rangle+\lambda\left\langle u_{m}(\tau), v(\tau)\right\rangle\right) d \tau \\
& +\int_{\mathbf{0}}^{t}\left\langle\sum_{i, j=1, \ldots, l} a_{i j} \frac{\partial}{\partial x_{j}} u_{m}, \frac{\partial}{\partial x_{i}} v\right\rangle d \tau+\int_{0}^{t}\langle b, v\rangle d \tau=\int_{0}^{t}\langle f, v\rangle d \tau .
\end{aligned}
$$

The $\wp_{m}$ is the set of functions $u_{m}$ and $\wp=\bigcup_{m} \wp_{m}$, the set $\wp$ is dense in $W_{1}^{2}$. Passing to the limit as $m \rightarrow \infty$ we obtain:

$$
\begin{aligned}
& \int_{0}^{t}\left(-\left\langle u(\tau), \partial_{t} v(\tau)\right\rangle+\lambda\langle u(\tau), v(\tau)\rangle\right) d \tau \\
& +\int_{0}^{t}\left\langle\sum_{i, j=1, \ldots, l} a_{i j} \frac{\partial}{\partial x_{j}} u, \frac{\partial}{\partial x_{i}} v\right\rangle d \tau \\
& +\int_{0}^{t}\langle b, v\rangle d \tau+\left.\langle u(\tau), v(\tau)\rangle\right|_{0} ^{t}=\int_{0}^{t}\langle f, v\rangle d \tau
\end{aligned}
$$

for any function $v \in \wp$.

Let us assume $v=u_{m}-\varphi$ then we have:

$$
\begin{aligned}
& \left.\left\langle u_{m}(\tau), u_{m}-\varphi\right\rangle\right|_{0} ^{t} \\
& +\int_{0}^{t}\left(\begin{array}{l}
\left.-\left\langle u_{m}(\tau), \partial_{t}\left(u_{m}-\varphi\right)(\tau)\right\rangle+\right) d \tau \\
+\lambda\left\langle u_{m}(\tau),\left(u_{m}-\varphi\right)(\tau)\right\rangle
\end{array}\right) \\
& +\int_{0}^{t}\left\langle\sum_{i, j=1, \ldots, l} a_{i j} \frac{\partial}{\partial x_{j}} u_{m}, \frac{\partial}{\partial x_{i}}\left(u_{m}-\varphi\right)\right\rangle d \tau \\
& +\int_{0}^{t}\left\langle b,\left(u_{m}-\varphi\right)\right\rangle d \tau=\int_{0}^{t}\left\langle f,\left(u_{m}-\varphi\right)\right\rangle d \tau
\end{aligned}
$$

SO:

$$
\begin{aligned}
& \int_{0}^{t}\left\langle\sum_{i, j=1, \ldots, l} a_{i j} \frac{\partial}{\partial x_{j}} u_{m}, \frac{\partial}{\partial x_{i}}\left(u_{m}-\varphi\right)\right\rangle d \tau \\
& =-\left(\begin{array}{l}
\left.\left\langle u_{m}(\tau), u_{m}-\varphi\right\rangle\right|_{0} ^{t}+ \\
+\int_{0}^{t}\left(\begin{array}{l}
-\left\langle u_{m}(\tau), \partial_{t}\left(u_{m}-\varphi\right)(\tau)\right\rangle+ \\
+\lambda\left\langle u_{m}(\tau),\left(u_{m}-\varphi\right)(\tau)\right\rangle
\end{array}\right) d \tau+ \\
+\int_{0}^{t}\left\langle b,\left(u_{m}-\varphi\right)\right\rangle d \tau
\end{array}\right) \\
& +\int_{0}^{t}\left\langle f,\left(u_{m}-\varphi\right)\right\rangle d \tau
\end{aligned}
$$

and: 


$$
\begin{aligned}
& \int_{0}^{t}\left(\begin{array}{l}
\left.-\left\langle u_{m}(\tau), \partial_{t}\left(u_{m}-\varphi\right)(\tau)\right\rangle+\right) d \tau \\
+\lambda\left\langle u_{m}(\tau),\left(u_{m}-\varphi\right)(\tau)\right\rangle
\end{array}\right) d \tau \\
& +\int_{0}^{t}\left\langle\sum_{i, j=1, \ldots, l} a_{i j} \frac{\partial}{\partial x_{j}} u_{m}, \frac{\partial}{\partial x_{i}}\left(u_{m}-\varphi\right)\right\rangle d \tau \\
& +\int_{\mathbf{0}}^{t}\left\langle b,\left(u_{m}-\varphi\right)\right\rangle d \tau-\int_{\mathbf{0}}^{t}\left\langle f,\left(u_{m}-\varphi\right)\right\rangle d \tau \\
& -\frac{\mathbf{1}}{\mathbf{2}}\left\|u_{m}\right\|^{2} \|_{t=\mathbf{0}}^{t}+\left.\left(u_{m}, \varphi\right)\right|_{\substack{t=0 \\
t=t}} ^{t=t} \text { function }\left(\left\|u_{m}-\varphi\right\|\right) \geq \mathbf{0},
\end{aligned}
$$

we fix the function $\varphi$ and pass to the limit as $m \rightarrow \infty$ obtain:

$$
\begin{aligned}
& \int_{0}^{t}\left(-\left\langle u(\tau), \partial_{t}(u-\varphi)(\tau)\right\rangle+\lambda\langle u(\tau),(u-\varphi)(\tau)\rangle\right) d \tau \\
& +\int_{0}^{t}\left\langle\sum_{i, j=1, \ldots, l} a_{i j} \frac{\partial}{\partial x_{j}} u, \frac{\partial}{\partial x_{i}}(u-\varphi)\right\rangle d \tau \\
& +\int_{0}^{t}\langle b,(u-\varphi)\rangle d \tau \\
& -\int_{0}^{t}\langle f,(u-\varphi)\rangle d \tau-\left.\frac{\mathbf{1}}{\mathbf{2}}\|u\|^{2}\right|_{t=0} ^{t=t} \\
& +\left.(u, \varphi)\right|_{t=0} ^{t=t}+\text { function }(\|u-\varphi\|) \geq \mathbf{0} .
\end{aligned}
$$

In the last inequality, we put $v=u$ and have:

$$
\begin{aligned}
& \left.\frac{\mathbf{1}}{\mathbf{2}}\|u\|^{2}\right|_{0} ^{t}+\lambda \int_{\mathbf{0}}^{t}\|u(\tau)\|^{2} d \tau \\
& +\int_{0}^{t}\left\langle\sum_{i, j=1, \ldots, l} a_{i j} \frac{\partial}{\partial x_{j}} u, \frac{\partial}{\partial x_{i}} u\right\rangle d \tau+\int_{0}^{t}\langle b, u\rangle d \tau=\int_{0}^{t}\langle f, u\rangle d \tau .
\end{aligned}
$$

Since $v \in \wp_{m}$ for arbitrary $m$ therefore for arbitrary function $v \in \wp=\bigcup_{m=1}^{\infty} \wp_{m}$, we have:

$$
\begin{aligned}
& \int_{0}^{t}\left(-\left\langle u(\tau), \partial_{t}(u-v)(\tau)\right\rangle+\lambda\langle u(\tau),(u-v)(\tau)\rangle\right) d \tau \\
& +\int_{0}^{t}\left\langle\sum_{i, j=1, \ldots, l} a_{i j} \frac{\partial}{\partial x_{j}} u, \frac{\partial}{\partial x_{i}}(u-v)\right\rangle d \tau+\int_{0}^{t}\langle b,(u-v)\rangle d \tau \\
& -\int_{0}^{t}\langle f, u-v\rangle d \tau+\text { function }(\|u-v\|) \geq \mathbf{0} .
\end{aligned}
$$

Since the set $\wp$ is dense in $W_{1}^{2}$ therefore for any $\varepsilon>0$ and any function $\varphi \in \wp$, we can put $v=u-\varepsilon \varphi$ and estimate:

$$
\begin{aligned}
& \varepsilon \int_{0}^{t}\left(-\left\langle u(\tau), \partial_{t} \varphi(\tau)\right\rangle+\lambda\langle u(\tau), \varphi(\tau)\rangle\right) d \tau \\
& +\varepsilon \int_{0}^{t}\left\langle\sum_{i, j=1, \ldots, l} a_{i j} \frac{\partial}{\partial x_{j}} u, \frac{\partial}{\partial x_{i}} \varphi\right\rangle d \tau+\varepsilon \int_{0}^{t}\langle b, \varphi\rangle d \tau \\
& -\varepsilon \int_{0}^{t}\langle f, \varphi\rangle d \tau+\text { function }(\varepsilon\|\varphi\|) \geq \mathbf{0} .
\end{aligned}
$$

We pass to the limit as $\varepsilon \rightarrow 0$ have:

$$
\begin{aligned}
& \int_{0}^{t}\left(-\left\langle u(\tau), \partial_{t} \varphi(\tau)\right\rangle+\lambda\langle u(\tau), \varphi(\tau)\rangle\right) d \tau \\
& +\int_{0}^{t}\left\langle\sum_{i, j=1, \ldots, l} a_{i j} \frac{\partial}{\partial x_{j}} u, \frac{\partial}{\partial x_{i}} \varphi\right\rangle d \tau+\int_{0}^{t}\langle b, \varphi\rangle d \tau-\int_{0}^{t}\langle f, \varphi\rangle d \tau \geq \mathbf{0} .
\end{aligned}
$$

Since the set $\wp$ is dense in $W_{1}^{2}$, from the last inequality, the estimation:

$$
\begin{aligned}
& \int_{0}^{t}\left(-\left\langle u(\tau), \partial_{t} \varphi(\tau)\right\rangle+\lambda\langle u(\tau), \varphi(\tau)\rangle\right) d \tau \\
& +\int_{0}^{t}\left\langle\sum_{i, j=1, \ldots, l} a_{i j} \frac{\partial}{\partial x_{j}} u, \frac{\partial}{\partial x_{i}} \varphi\right\rangle d \tau+\int_{0}^{t}\langle b, \varphi\rangle d \tau-\int_{0}^{t}\langle f, \varphi\rangle d \tau=\mathbf{0},
\end{aligned}
$$

is true for arbitrary $\varphi \in W_{1}^{2}$, which means that function $u \in W_{1}^{2}$ is a solution to (1).

\section{Remark}

The monotonousness can be proven as:

$$
\begin{aligned}
& \int_{0}^{t}\left(\begin{array}{l}
-\left\langle u_{m}(\tau)-v(\tau), \partial_{t}\left(u_{m}(\tau)-v(\tau)\right)\right\rangle+ \\
+\lambda\left\langle u_{m}(\tau)-v(\tau), u_{m}(\tau)-v(\tau)\right\rangle
\end{array}\right) d \tau \\
& +\int_{0}^{t}\left(\sum_{i, j=1, \ldots, l}\left(\begin{array}{l}
a_{i j}\left(\tau, x, u_{m}\right) \frac{\partial}{\partial x_{j}} u_{m}- \\
-a_{i j}(\tau, x, v) \frac{\partial}{\partial x_{j}} v, \frac{\partial}{\partial x_{i}}\left(u_{m}(\tau)-v(\tau)\right)
\end{array}\right)\right) d \tau \\
& +\int_{0}^{t}\left\langle b\left(\tau, x, u_{m}, \nabla u_{m}\right)-b(\tau, x, v, \nabla v), u_{m}(\tau)-v(\tau)\right\rangle d \tau \\
& +\left.\left\langle u_{m}(\tau)-v(\tau), u_{m}(\tau)-v(\tau)\right\rangle\right|_{0} ^{t} \\
& \geq \int_{0}^{t}\left(\begin{array}{l}
\left.-\left\langle u_{m}(\tau)-v(\tau), \partial_{t}\left(u_{m}(\tau)-v(\tau)\right)\right\rangle+\right) \\
+\lambda\left\|u_{m}(\tau)-v(\tau)\right\|^{2}
\end{array}\right) d \tau \\
& +\int_{0}^{t}\left(\begin{array}{l}
\sum_{i, j=1, \ldots, l} a_{i j}\left(\tau, x, u_{m}\right) \frac{\partial}{\partial x_{j}} u_{m}- \\
-\sum_{i, j=1, \ldots, l} a_{i j}(\tau, x, v) \frac{\partial}{\partial x_{j}} v, \frac{\partial}{\partial x_{i}}\left(u_{m}(\tau)-v(\tau)\right)
\end{array}\right) d \tau \\
& +\int_{0}^{t}\left\langle\begin{array}{l}
b\left(\tau, x, u_{m}, \nabla u_{m}\right)-b(\tau, x, v, \nabla v), u_{m}(\tau)- \\
-v(\tau)
\end{array}\right) d \tau \\
& +\left.\left\|u_{m}(\tau)-v(\tau)\right\|^{2}\right|_{0} ^{t} \\
& \geq \int_{0}^{t}\left(-\left\langle w, \partial_{t} w\right\rangle+\lambda\|w\|^{2}\right) d \tau \\
& +\int_{0}^{t}\left(\begin{array}{l}
\sum_{i, j=1, \ldots, l} a_{i j}\left(\tau, x, u_{m}\right) \frac{\partial}{\partial x_{j}} u_{m}- \\
-\sum_{i, j=1, \ldots, l} a_{i j}(\tau, x, v) \frac{\partial}{\partial x_{j}} v, \frac{\partial}{\partial x_{i}} w
\end{array}\right) d \tau
\end{aligned}
$$




$$
\begin{aligned}
& -\frac{\mathbf{1}}{p}\left(\frac{\mathbf{1}}{\varepsilon^{2}}\|\nabla w\|^{2}+\varepsilon^{2}\left(\beta\langle\nabla w \circ a \circ \nabla w\rangle+c(\beta)\|w\|^{2}\right)\right) \\
& -\beta\langle\nabla w \circ a \circ \nabla w\rangle+c(\beta)\|w\|^{2}+\left.\|w(\tau)\|^{2}\right|_{0} ^{t},
\end{aligned}
$$

since

$$
\left|b\left(t, x, u_{m}, \nabla u_{m}\right)-b(t, x, v, \nabla v)\right| \leq \mu_{4}(x)\left|\nabla\left(u_{m}-v\right)\right|
$$

$+\mu_{5}(x)\left|u_{m}-v\right|$ and we had denoted $w=\left(u_{m}-v\right)$ and estimated:

$$
\begin{aligned}
& \frac{\mathbf{2}}{p}\left\langle\mu_{4}(x)|\nabla w|,|w|\right\rangle \\
& \leq \frac{\mathbf{2}}{p}\left\|\mu_{4} w\right\|\|\nabla w\|=\frac{\mathbf{2}}{p}\|\nabla w\|\left\langle\left(\mu_{4} w\right)^{2}\right\rangle^{\frac{1}{2}} \\
& \leq \frac{\mathbf{2}}{p}\|\nabla w\|\left(\beta\langle\nabla w \circ a \circ \nabla w\rangle+c(\beta)\|w\|^{2}\right)^{\frac{1}{2}} \\
& \leq \frac{\mathbf{1}}{p}\left(\frac{\mathbf{1}}{\varepsilon^{2}}\|\nabla w\|^{2}+\varepsilon^{2}\left(\beta\langle\nabla w \circ a \circ \nabla w\rangle+c(\beta)\|w\|^{2}\right)\right),
\end{aligned}
$$

and:

$$
\begin{aligned}
& \left\langle\mu_{5}(x)|u-v|,(u-v)\right\rangle=\left\langle\mu_{5}, w^{2}\right\rangle \\
& \leq \beta\langle\nabla w \circ a \circ \nabla w\rangle+c(\beta)\|w\|^{2} .
\end{aligned}
$$

\section{The Regularity of the Solution to the Cauchy Problem for the Parabolic Equation (1)}

\section{Theorem 3}

Assume that there is a sequence of parabolic partial differential equations:

$$
\begin{aligned}
& \frac{\partial}{\partial t} u+\lambda^{z} u-\frac{\partial}{\partial x_{i}}\left(a_{i j}^{z}(t, x) \frac{\partial}{\partial x_{j}} u\right) \\
& +b^{z}(t, x, u, \nabla u)=f^{z}(t, x), \quad z=\mathbf{1}, 2, \ldots . .
\end{aligned}
$$

and each equation satisfies the conditions of the existence of the solution (1) with the same coefficients' restrictions for all values of the parameter $z=\mathbf{1}, \mathbf{2}, \ldots . .$. . Let us denote the sequence of the weak solutions $u^{z} \in V_{\mathbf{1 , 0}}^{\mathbf{2}}, \quad z=\mathbf{1}, \mathbf{2}, \ldots$. to the Cauchy problems for the Equations (15) under initial conditions $u^{z}(\mathbf{0}, x) \equiv \varphi_{0}{ }^{2}$. Let the conditions:

$$
\begin{aligned}
& \lim _{z \rightarrow \infty}\left\|u_{\mathbf{0}}-\varphi_{\mathbf{0}}{ }^{z}\right\|=\mathbf{0}, \\
& \lim _{z \rightarrow \infty}\left\|\sum_{i, j=1, \ldots, l, l}\left(a_{i j}-a_{i j}{ }^{z}\right)\right\|=\mathbf{0} ; \\
& \lim _{z \rightarrow \infty} \int_{\mathbf{0}}^{t}\left\langle f(\tau, \cdot)-f^{z}(\tau, \cdot), \eta\right\rangle d \tau=\mathbf{0} ; \\
& \lim _{z \rightarrow \infty} \int_{0}^{t}\left\langle\left|b(\tau, \cdot, u, \nabla u)-b^{z}(\tau, \cdot, u, \nabla u)\right|, \eta\right\rangle d \tau=\mathbf{0},
\end{aligned}
$$

are satisfied, these equations mean that the coefficient of (15) converge to the coefficients (1) and additional condition:

$$
\begin{aligned}
& \left|b^{z}(\tau, \cdot, u, \nabla u)-b^{z}\left(\tau, \cdot, u^{z}, \nabla u^{z}\right)\right| \\
& \leq \mu_{4}(\cdot)\left|\nabla\left(u-u^{z}\right)\right|+\mu_{5}(\cdot)\left|u-u^{z}\right|
\end{aligned}
$$

is executed.

Then the sequence of the weak solution $u^{z} \in V_{\mathbf{1}, \mathbf{0}}^{\mathbf{2}}$, $z=\mathbf{1}, \mathbf{2}, \ldots .$. to the Cauchy problems for the equations (15) under the initial conditions $u^{z}(\mathbf{0}, x)=\varphi_{0}{ }^{2}$ converges to the weak solution to the Cauchy problem for the equation (1) under the initial condition $u(\mathbf{0}, x)=u_{\mathbf{0}}$ in $V_{\mathbf{1 , 0}}^{\mathbf{2}}$.

Proof

The proving will be accomplished according to the schema:

- compose the integral identity for the solution $u(t, x)$ to the Cauchy problem for the equation (1) under the initial condition $u(0, x)=u_{0}$ and for the sequence of the weak solutions $u^{z} \in V_{10}^{2}, z=\mathbf{1}, 2, \ldots .$. to the Cauchy problems for the equations (15) under the initial conditions $u^{z}(\mathbf{0}, x)=\varphi_{0}{ }^{z}$

- $\quad$ subtract integral identity for the solution $u^{z} \in V_{\mathbf{1 0}}^{2}$, $z=\mathbf{1}, 2, \ldots .$. from the integral identity for the solution $u(t, x)$, the results of these subtractions are written as the integral identity for the differences $v^{z}=u-u^{z}$

- $\quad$ obtain the priory estimations for the differences $v^{z}=$ $u-u^{z}$

- apply the priory estimations to substantiate the passing to the $\operatorname{limit} \lim v^{z}=\mathbf{0}$ in $V_{\mathbf{1 , 0}}^{2}$ topology.

Let us compose the integral identity for the (1):

$$
\begin{aligned}
& \left.\langle u(\tau), \eta(\tau)\rangle\right|_{0} ^{t}+\int_{0}^{t}\left(-\left\langle u(\tau), \partial_{t} \eta(\tau)\right\rangle+\lambda\langle u(\tau), \eta(\tau)\rangle\right) d \tau \\
& +\int_{0}^{t}\left\langle\sum_{i, j=1, \ldots, l} a_{i j} \frac{\partial}{\partial x_{j}} u, \frac{\partial}{\partial x_{i}} \eta\right\rangle d \tau+\int_{0}^{t}\langle b, \eta\rangle d \tau=\int_{0}^{t}\langle f, \eta\rangle d \tau
\end{aligned}
$$

for an arbitrary $\eta \in W_{\mathbf{1 . 0}}^{q}$ and the integral identities for the Equations (15):

$$
\begin{aligned}
& \left.\left\langle u^{z}(\tau), \eta(\tau)\right\rangle\right|_{0} ^{t}+\int_{0}^{t}\left(-\left\langle u^{z}(\tau), \partial_{t} \eta(\tau)\right\rangle+\lambda^{z}\left\langle u^{z}(\tau), \eta(\tau)\right\rangle\right) d \tau \\
& +\int_{0}^{t}\left\langle\sum_{i, j=1, \ldots, l} a_{i j}{ }^{z}(\tau, \cdot) \frac{\partial}{\partial x_{j}} u^{z}, \frac{\partial}{\partial x_{i}} \eta\right\rangle d \tau \\
& +\int_{0}^{t}\left\langle b^{z}\left(\tau, \cdot, u^{z}, \nabla u^{z}\right), \eta\right\rangle d \tau=\int_{0}^{t}\left\langle f^{z}(\tau, \cdot), \eta\right\rangle d \tau
\end{aligned}
$$


for an arbitrary $\eta \in W_{\mathbf{1 . 0}}^{q}$, after the subtraction, we are obtaining the equation:

$$
\begin{aligned}
& \left.\left\langle v^{z}(\tau), \eta(\tau)\right\rangle\right|_{0} ^{t} \\
& +\int_{0}^{t}\left(-\left\langle v^{z}(\tau), \partial_{t} \eta(\tau)\right\rangle+\lambda\left\langle v^{z}(\tau), \eta(\tau)\right\rangle\right) d \tau \\
& +\int_{0}^{t}\left\langle\sum_{i, j=1, \ldots, l}\left(a_{i j}(\tau, \cdot)-a_{i j}{ }^{2}(\tau, \cdot)\right) \frac{\partial}{\partial x_{j}} u, \frac{\partial}{\partial x_{i}} \eta\right\rangle d \tau \\
& +\int_{0}^{t}\left\langle\sum_{i, j=1, \ldots, l} a_{i j}{ }^{z}(\tau, \cdot) \frac{\partial}{\partial x_{j}} v^{z}, \frac{\partial}{\partial x_{i}} \eta\right\rangle d \tau \\
& +\int_{0}^{t}\left\langle b(\tau, \cdot, u, \nabla u)-b^{z}\left(\tau, \cdot, u^{z}, \nabla u^{z}\right), \eta\right\rangle d \tau \\
& =\int_{0}^{t}\left\langle f(\tau, \cdot)-f^{z}(\tau, \cdot), \eta\right\rangle d \tau .
\end{aligned}
$$

$$
\begin{aligned}
& \text { Let us estimate the } \\
& \int_{0}^{t}\left\langle\sum_{i, j=1, \ldots, l}\left(a_{i j}(\tau, \cdot)-a_{i j}{ }^{z}(\tau, \cdot)\right) \frac{\partial}{\partial x_{j}} u, \frac{\partial}{\partial x_{i}} \eta\right\rangle d \tau \text {, since: } \\
& \lim _{z \rightarrow \infty}\left\|\sum_{i, j=1, \ldots, l} a_{i j}(\tau, x)-a_{i j}^{z}(\tau, x)\right\| \\
& =\lim _{z \rightarrow \infty} \sqrt{\int_{0}^{t}\left\langle\sum_{i, j=1, \ldots, l}\left|a_{i j}(\tau, \cdot)-a_{i j}{ }^{2}(\tau, \cdot)\right|^{2}\right\rangle d \tau}=\mathbf{0}
\end{aligned}
$$

term

therefore:

$$
\lim _{z \rightarrow \infty} \int_{\mathbf{0}}^{t}\left\langle\sum_{i, j=\mathbf{1}, \ldots, l}\left(a_{i j}(\tau, \cdot)-a_{i j}{ }^{2}(\tau, \cdot)\right) \frac{\partial}{\partial x_{j}} u, \frac{\partial}{\partial x_{i}} \eta\right\rangle d \tau=\mathbf{0},
$$

applying the notation $v^{z}=u-u^{z}$ and fact $v^{z} \in W_{\mathbf{1 , 0}}^{\mathbf{2}}$, we have had:

$$
\begin{aligned}
& \left|\int_{\mathbf{0}}^{t}\left\langle\sum_{i, j=\mathbf{1}, \ldots, l} a_{i j}{ }^{z}(\tau, \cdot) \frac{\partial}{\partial x_{j}} v^{z}, \frac{\partial}{\partial x_{i}} \eta\right\rangle d \tau\right| \\
& \leq\left\|\sum_{i, j=\mathbf{1}, \ldots, l} a_{i j}{ }^{2} \frac{\partial}{\partial x_{i}} \eta\right\|\left\|\mid \frac{\partial}{\partial x_{j}} v^{z}\right\|
\end{aligned}
$$

From the conditions we have:

$$
\lim _{z \rightarrow \infty} \int_{\mathbf{0}}^{t}\left\langle f(\tau, \cdot)-f^{z}(\tau, \cdot), \eta\right\rangle d \tau=\mathbf{0},
$$

Since:

$$
\lim _{z \rightarrow \infty} \int_{0}^{t}\left\langle\left|b(\tau, \cdot, u, \nabla u)-b^{z}(\tau, \cdot, u, \nabla u)\right|, \eta\right\rangle d \tau=\mathbf{0},
$$

and $\eta=v^{z}$, we obtain:

$$
\begin{aligned}
& \left\langle\left|b^{z}(\tau, \cdot, u, \nabla u)-b^{z}\left(\tau, \cdot, u^{z}, \nabla u^{z}\right)\right|, v^{z}\right\rangle \\
& \leq\left\|\mu_{4} v^{z}\right\|\left\|\nabla v^{z}\right\|+\left\|\mu_{5} v^{z}\right\|\left\|v^{z}\right\| \\
& \leq \frac{\mathbf{1}}{\mathbf{2}}\left(\frac{\mathbf{1}}{\sigma^{2}}\left\|\mu_{4} v^{z}\right\|^{2}+\sigma^{2}\left\|\nabla v^{z}\right\|^{2}\right) \\
& +\frac{\mathbf{1}}{\mathbf{2}}\left(\frac{\mathbf{1}}{\zeta^{2}}\left\|\mu_{5} v^{z}\right\|^{2}+\zeta^{2}\left\|v^{z}\right\|^{2}\right)
\end{aligned}
$$

so:

$$
\left\|\mu_{4} v^{z}\right\|^{2}=\left\langle v^{z}\left(\mu_{4}\right)^{2} v^{z}\right\rangle \leq \beta\left\|\nabla v^{z}\right\|^{2}+c(\beta)\left\|v^{z}\right\|^{2}
$$

similarly, the term containing $\mu_{5}$ can be estimated. After reducing similar terms, we obtain the statement of the theorem.

\section{Ethics}

This article is original and contains unpublished material. The corresponding author confirms that all of the other authors have read and approved the manuscript and no ethical issues involved.

\section{References}

Adams, D.R. and L.I. Hedberg, 1996. Function Spaces and Potential Theory. 1st Edn., Springer-Verlag, Berlin, ISBN-10: 3540570608, pp: 366.

DeGiorgi, E., 1968. Un esempio di estremali discontinue per un problema variazionale di tipo ellitico, Bull. UMI, 4: 135-137. [D-H-K-W] Dierkes, U., S. Hildebrandt, A. Kuster and O. Wohlrab, 1992. Minimal Surfaces. Springer-Verlag, Berlin.

Fijavz, M.K., D. Mugnolo and E. Sikolya, 2007. Variational and semigroup methods for waves and diffusion in networks. Applied Math. Optim., 55: 219-240. DOI: 10.1007/s00245-006-0887-9

Gilbarg, D. and N.S. Trudinger, 1983. Elliptic Partial Differential Equations of Second Order. 2nd Ed., Springer-Verlag, Berlin.

Ladyzenskaja, O.A., V.A. Solonnikov and N.N. Ural'ceva, 1968. Linear and Quasi-Linear equations of Parabolic type.

Nirenberg, L., 1994. Partial differential equations in the first half of the century. In: Development of Mathematics 1900-1950, Pier, J.P. (Ed.), Birkha User, Basel. 
Mykola Yaremenko / Journal of Mathematics and Statistics 2020, Volume 16: 76.89 DOI: $10.3844 /$ jmssp.2020.76.89

Veron, L., 1996. Singularities of Solutions of SecondOrder Quasilinear Equations. 1st Edn., CRC Press, Longman, Harlow New York, ISBN-10: 0582035392, pp: 392.

Yaremenko, M.I., 2017a. The existence of a solution of evolution and elliptic equations with singular coefficients. Asian J. Math. Comput. Res., 15: 172-204.
Yaremenko, M.I., 2017b. Quasi-linear evolution and elliptic equations. J. Progressive Res. Math., 11: 1645-1669. 